\title{
Vier Szenarien für die Hochschule der Zukunft
}

Im vorangegangenen Kapitel (Kapitel C.1) haben wir gezeigt, welche Einflussfaktoren sich Hochschulen heute gegenübersehen. Aus den genannten Faktoren konnten in den Interviewdaten der NextSkills Studie Hinweise rekonstruiert werden, die auf vier Entwicklungsstränge hindeuten, die dominant auf die zukünftige Entwicklung von Hochschulen wirken werden (Kapitel C 3.1 Entwicklungsrahmen für die Hochschule der Zukunft: Vier Säulen für den Hochschulwandel). Diese wurden im Rahmen des NextSkills Delphi Expertinnen und Experten vorgelegt, um sie diskursiv validieren zu lassen. Dabei wurden die Expertinnen und Experten gebeten, zwei Dinge zu tun: Zum einen sollten sie einschätzen, wie sie die Relevanz der jeweils formulierten Einflussfaktoren bewerteten. Zum anderen sollten sie auch die sogenannte time to adoption einschätzen, also die Zeitspanne, in der aus ihrer Sicht die Einflussfaktoren in den Hochschulen tatsächlich Gestalt annähmen und umgesetzt werden würden.

Den Befragten wurden auf Basis dieser Einflussfaktoren zusätzlich noch vier Zukunftsszenarien vorgelegt. Diese wurden auf der Grundlage eines Basisszenarios konstruiert (Business as usual Szenario) welches in jedem Szenario dann um die Annahme der Realisierung eines der vier Einflussfaktoren erweitert wurde.

\section{3.1 Entwicklungsrahmen für die Hochschule der Zukunft: Vier Säulen für den Hochschulwandel}

Die Studienergebnisse weisen auf vier verschiedene Einflussfaktoren hin, die im Folgenden als die Säulen des Wandels in der Hochschulbildung bezeichnet werden. Die Verwendung des Begriffs Säulen ist durchaus im Sinne einer raumarchitektonischen Metapher gemeint, die ausdrücken soll, dass hier ein Raum aufgespannt wird, in welchem sich Hochschulbildung in den nächsten zehn bis fünfzehn Jahren entwickeln wird (siehe Abbildung 49). Die Identifikation dieser Faktoren ist 


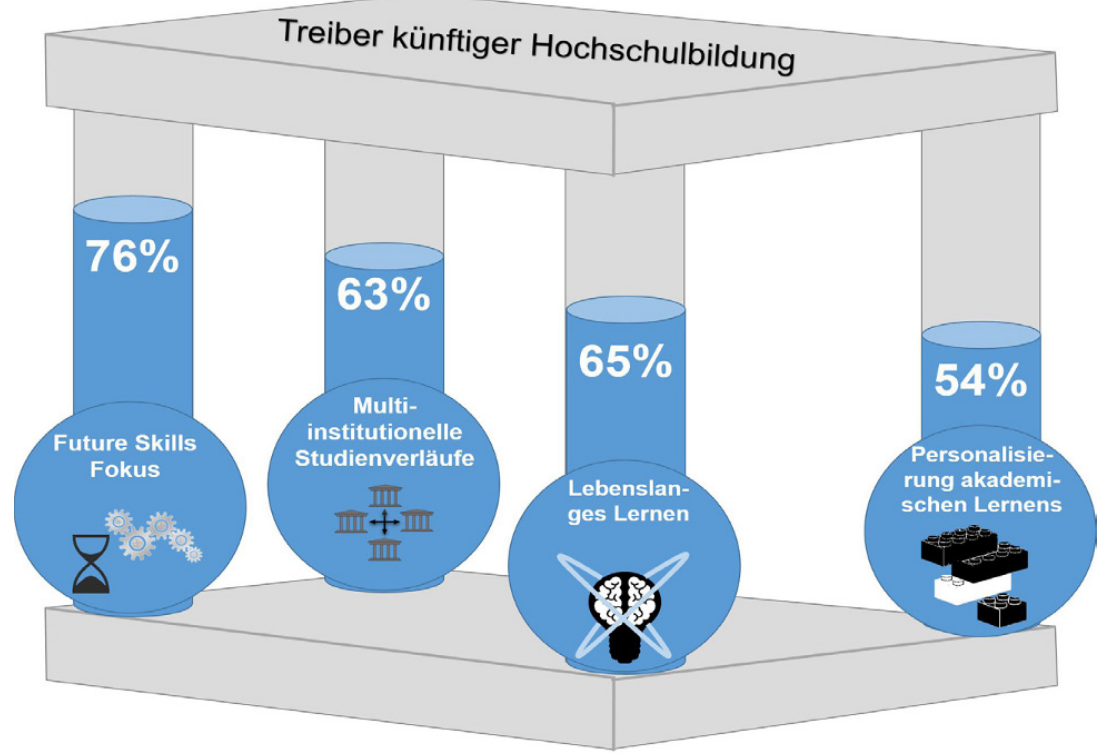

Abb. 49 Der vier Säulenraum des Delphis für die zukünftige Entwicklung der Hochschulbildung mit Zustimmungswerten des Samples ${ }^{47}(\mathrm{~N}=46)$

methodisch der erste Schritt im Rahmen einer Szenariomethode, in der dann in Kapitel C 3.2 Szenarien für die Hochschulentwicklung identifiziert werden. Zwar ist es nicht möglich Umfang, Intensität und Geschwindigkeit des Wandels exakt einzugrenzen, jedoch kann so über die Gestalt des Wandels Auskunft gegeben werden. Für die nachfolgend dargestellten vier Säulen des Wandels ergaben sich hohe Zustimmungswerte (siehe Prozentwerte innerhalb der Säulen).

Das Delphiverfahren erlaubt es als expertenorientiertes konsensuales Verfahren, dass Expertinnen und Experten zunächst in der ersten Delphirunde die Formulierungen anpassten und Bestandteile hinzufügten oder änderten sowie Gewichtungen der Formulierungen veränderten. Sie konnten ihre Zustimmung zu den vier Faktoren auf einer fünf-stufigen Likert-Skala angeben, die zwischen den beiden Polen $1=$ „starke Ablehnung“ und $5=$,starke Zustimmung“ rangierte. Für den Fall, dass sie einen Faktor kommentieren wollten - zum Beispiel zum Zweck

47 Der Zustimmungswert A (für Agreement index) gibt den prozentualen Anteil derjenigen an, die mit 4 oder 5 (Zustimmung bzw. starke Zustimmung) auf der 5-stufigen Likert Skala geantwortet haben. 
der Item-Reformulierung oder als Spezifikation/ Erklärung für ihr Antwortverhalten - wurde ein separates Kommentarfeld zur Verfügung gestellt. Auf diese Weise konnten etwa 26 Hinweise und Kommentare gesammelt werden, mit der die Formulierungstiefe und -reichweite verbessert werden konnte. In der zweiten Runde wurden diese genaueren Formulierungen dann noch einmal von allen teilnehmenden Expertinnen und Experten hinsichtlich der Zeitspanne eingeschätzt, in der diese Einflussfaktoren in der Hochschule umgesetzt werden würden. Im Folgenden werden diese als die tragenden Säulen beschrieben, die den Entwicklungsraum für die zukünftige Hochschulbildung aufspannen (siehe Abbildung 49). Die hier genutzte architektonische Metapher eines Entwicklungsraumes verweist explizit darauf, dass es ein Zusammenspiel aller vier Faktoren sein wird, welches die Gestalt der zukünftigen Hochschulprofile bestimmen wird.

\section{3.1.1 Säule 1: Future Skills Fokus}

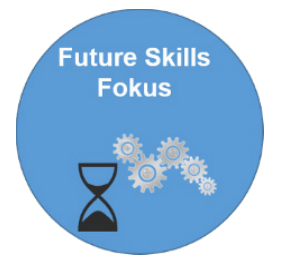

Ein aufkommender Future Skills-Fokus verändert den in der Hochschulbildung bislang geläufigen Fokus der Wissensvermittlung zugunsten eines „next mode“ des Studierens.

Faktor 1 zufolge wird sich der Fokus in der Hochschulbildung auf Future Skills verlagern, was zu einem radikalen Wandel der bisherigen Definition der Attribute von Absolventinnen und Absolventen führen wird. Dieser Wandel beinhaltet, dass sich der momentane Fokus in der Hochschulbildung von akademischem und validem Fachwissen (Lernen wird als Mittel verstanden, um richtige Antworten auf bekannte Fragestellungen geben zu können) zugunsten eines next modes des Studierens wandeln würde. Lernen wird in diesem next mode als Anwendung und Reflexion über Wissen und als kreatives Entwickeln von neuem Wissen verstanden, was an die Stelle des Auswendiglernens von Fachwissen tritt. Um dies zu unterstützen, kämen neue Lehr- und Lernmethoden zur Anwendung, welche auf die Entwicklung von Future Skills abzielten. Das internationale Delphi-Panel stimmte diesem Faktor generell zu $\left(M=3,81, S D=1,22, A_{\text {Factorl }}=76,1 \%\right) .{ }^{48}$ Dank der qualitativen Kommentare der

48 A Factor 1 bezeichnet die Zustimmung (A=Agreement) zu Faktor 1 und gibt den prozentualen Anteil derjenigen an, die mit 4 oder 5 (Zustimmung bzw. starke Zustimmung) auf der 5-stufigen Likert Skala geantwortet haben. 
Expertinnen und Experten konnte die Beschreibung des Faktors weiter in Richtung einer klareren und stärkeren Vision von Future Skills und Lernen verfeinert werden. Unter anderem zeigte sich, dass Future Skills und Wissen nicht als opponierende Bestandteile von Hochschulbildung wahrgenommen werden, sondern vielmehr aufeinander aufbauen. Dabei stellt Wissen die Basis für Future Skills dar, reicht alleine aber nicht mehr aus. Das Expert(inn)engremium verwendete Begriffe wie „specialized knowledge“ (spezialisiertes Wissen) oder "knowledge mode 2“ (Wissensmodus 2) als semantische Marker, um darauf hinzuweisen, dass ein Konzept für „next knowledge" unabdingbar sei. Diese Konzepte können zu Gibbons et al. (1994) in Bezug gesetzt werden, die in diesem Kontext von neuer und gemeinschaftlicher Wissens-produktion sprechen. Die Befragten gaben in einigen Fällen außerdem an, dass ein verstärkter Fokus auf Future Skills keine Zukunftsvision mehr sei, sondern in einigen Institutionen bereits zum festen Agenda-Bestandteil gehöre. Die Meinungen der Expertinnen und Experten zeichnen eine Fokusverschiebung nicht nur in Richtung eines veränderten Stellenwerts von Fachwissen nach, sondern zeigen außerdem die Wichtigkeit derjenigen Fähigkeiten auf, die hier als Future Skills bezeichnet werden. Während die Richtung und das Konzept der dargestellten Entwicklung sich in den Meinungen des Gremiums von Expertinnen und Experten klar abzeichnen, muss dennoch festgehalten werden, dass ein Future Skills Fokus nicht generalisierend für alle (Arten von) Hochschulen, Fakultäten, Fächer und Studienabschlüsse (Bachelor und Master) gleichermaßen erfolgskritisch sein wird.

\section{Future Skills: Geschätzte Zeitdauer für den Wandel}

Interessanterweise zeigte sich aus den Daten, dass der prognostizierte Wandel in Richtung eines stärkeren Fokussierens auf Future Skills in Hochschulen (Faktor 1) sich nach mehrheitlicher Meinung des internationalen Gremiums $(35,0 \%)$ bereits vollziehe. Autonome Lernkompetenz, Selbstorganisationskompetenz, Anwenden von und Reflexion über Fachwissen sowie Kreativität und Innovation seien bereits wichtige Bestandteile einer akademischen Ausbildung in vielen Hochschulen. Für diesen next mode des Studierens geht das Sample von Expertinnen und Experten davon aus, dass er nach und nach den reduzierten/ eingeschränkten Fokus auf akademischen Wissenserwerb (mit dem Ziel durch ein fixes Curriculum für ein bestimmtes Berufsfeld auszubilden) ersetzen wird. Abbildung 50 zeigt, dass die verbleibenden 65 Prozent davon ausgehen, dass die Wichtigkeit dieses Faktors sich in den kommenden Jahren und Jahrzehnten erhöhen wird. 


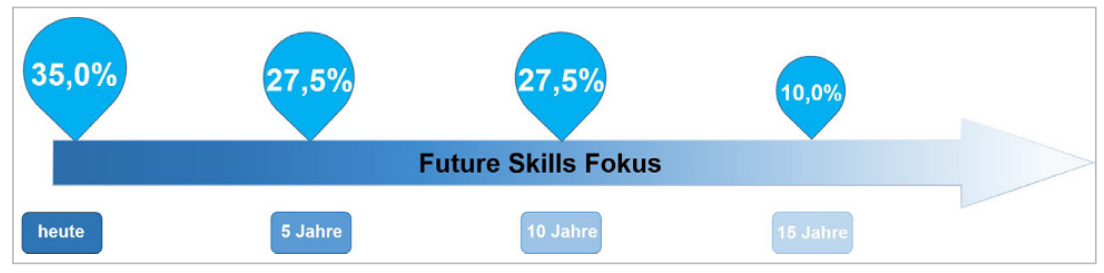

Abb. 50 Geschätzte Zeitdauer für die zunehmende Wichtigkeit eines Future Skills Fokus für Hochschulbildung $(\mathrm{N}=40)^{49}$

\section{3.1.2 Säule 2: Multi-institutionelle Studienverläufe}

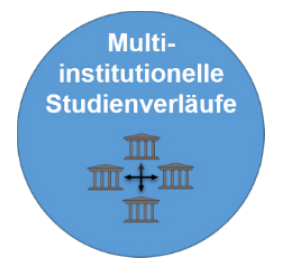

Hochschulbildung bewegt sich zunehmend von einem, ein-institutionellen hin zu einem, multi-institutionellen Modell, in dem sich mehrere Institutionen zu Hochschulbildung vermittelnden Allianzen verbinden.

Dem zweiten Faktor zufolge entwickelt sich Hochschulbildung zu einer multiinstitutionellen Studienerfahrung. Damit ist gemeint, dass Hochschulbildung tendenziell mit dem momentanen Ein-Institutionen-Modell brechen würde und sich stattdessen für Kooperationen mit anderen Institutionen in einem Allianzen-Netzwerk öffnen würde. Der Mittelwert von 3,72 (SD =1,12, $\left.A_{\text {Factor } 2}=63,0 \%\right)$ zeigt, dass das internationale Gremium von Expertinnen und Experten diesem Konzept generell zustimmt. Sie verweisen jedoch darauf, dass Studienverläufe, die sich über die institutionellen Grenzen hinweg erstrecken, eine fundiertere Praxis im Umgang mit der Anerkennung von bereits erbrachten Studienleistungen bedürften. Studierende würden in solch einem Setting ihre persönlichen Präferenzen der Reputation, Qualität und dem Angebot der Hochschulen entsprechend zwischen verschiedenen Institutionen wechseln. Kleinere oder größere Teile des Curriculums würden auf unterschiedliche Institutionen aufgeteilt, was eine Patchwork-artige, multi-institutionelle Studienorganisation bedeutete.

Während der Aspekt des Punktetransfers in den Bologna Unterzeichnerländern zumindest konzeptionell bereits real ist, herrscht auf institutioneller Ebene bislang

49 Anmerkung: In dieser und den folgenden Abbildungen kann es vorkommen, dass sich die Prozentwerte aufgrund von Rundungsfehlern zu mehr als 100 Prozent aufsummieren. 
noch mangelnde Praktikabilität diesbezüglich. Es zeigt sich, dass Fernhochschulen in diesem Punkt auf einen erheblich größeren Erfahrungsschatz zurückgreifen können, als die traditionellen Institutionen. Ein Delphi-Teilnehmer gab beispielsweise an, dass Studierende in Kanada bereits die Möglichkeit hätten, ihre Punkte zwischen Bildungsinstitutionen zu transferieren, wobei er die Vorreiterrolle der Canadian Virtual University besonders hervorhob. Zwei weitere Befragte erklärten, dass sich dieser Trend auch am Wahlverhalten der Studierenden im Hinblick auf die ausgesuchte Hochschule zeige: Studierende schrieben sich so beispielsweise für einen Bachelor-Studiengang an einer Hochschule ein, um dann im Anschluss für ihren Master eine andere Institution zu wählen. Erasmus mundus bietet beispielsweise einen gemeinsamen Masterabschluss an und organisiert die akademische Ausbildung der Studierenden als integrierten, internationalen Studiengang, der von einem Konsortium verschiedener Hochschulen bereitgestellt wird. Dieses Programm wurde vom Sample der Expertinnen und Experten ebenfalls als bereits existierendes Beispiel für die zunehmende Wichtigkeit multi-institutioneller Studienwege genannt. Ein Befragter spekulierte zudem, ob nicht das Internet als zusätzliche Quelle der Wissensgenerierung und -bereitstellung an die Stelle eines multi-institutionellen Netzwerks von Hochschulen treten könnte.

Während diese Beispiele demonstrieren, dass es bereits erste Evidenzen für multi-institutionelle Ansätze in der Hochschulbildung gibt, gaben drei Expertinnen und Experten an, dass dieser Trend ihrer Meinung nach erst in den nächsten fünf bis zehn Jahren real werden würde, stimmten dem generellen Trend hin zu dieser alternativen Form des Studienverlaufs jedoch zu.

Zudem versuchten die Expertinnen und Experten Gründe dafür zu identifizieren, warum Hochschulen in diese Art der Netzwerk-Organisation einsteigen sollten. Beispielsweise wurde ein Vorteil insbesondere für kleinere, spezialisierte Institutionen angeführt. Einer ähnlichen Argumentation folgend, äußerte sich einer der Experten kritisch im Hinblick auf die Bereitschaft großer Universitäten (wie beispielsweise die US-amerikanischen Ivy League Colleges) in solchen multi-institutionellen Arrangements zu partizipieren. Dies - so die Argumentation - könnte ihrem starken Markennamen schaden. Neben dem jeweiligen Charakter der unterschiedlichen Hochschulen und Hochschularten wurden außerdem regulatorische und ökonomische Rahmenbedingungen diskutiert, die entweder als ermöglichende oder beschränkende Bedingungen für die Formierung multi-institutioneller Netzwerke fungieren könnten. Zwei weitere Stimmen des Samples identifizierten die Studierenden als Schlüsseleinflussgröße, von der es abhängen werde, ob und in wie weit sich dieser Faktor in zukünftigen Bildungsszenarien verankern würde. 


\section{Multi-institutionelle Studienverläufe: Geschätzte Zeitdauer für den Wandel}

Der zweite Faktor multi-institutionelle Studienverläufe wird laut Einschätzungen der Expertinnen und Experten in den nächsten fünf $(30,8 \%)$ bis zehn Jahren $(30,8 \%)$ an Wichtigkeit in Hochschulen gewinnen (siehe Abbildung 51). Voraussetzung hierfür ist die oben schon angeführte Entwicklung von umfassenden Regeln und Erfahrungen für die Praxis im Umgang mit der Anerkennung von bereits erbrachten Studienleistungen. Kleinere oder größere Teile des Curriculums würden auf unterschiedliche Institutionen aufgeteilt, was eine Patchwork-artige, multi-institutionelle Studienorganisation bedeutete.

Während der Bologna-Prozess und europäische Qualifikations-Rahmenpläne eine erste solide Basis für multi-institutionelle Allianzen im Hochschulsektor geschaffen haben, ist die gegenseitige Anerkennung von Punkten, die nicht in der eigenen Hochschule erworben wurden, noch lange nicht Realität geworden - diese Position zeigt sich auch in den qualitativen Kommentaren der Expertinnen und Experten. Trotz der systemischen Natur dieses Faktors und der Tatsache, dass Hochschulen grenzspannende Transfersysteme, portable Punkte und gegenseitig verständliche und vertrauenswürdige Formate akademischer Leistungsnachweise entwickeln werden müssen, wird dieser Trend nach Schätzungen des Samples der Expertinnen und Experten in den kommenden fünf bis zehn Jahren prominenter werden.

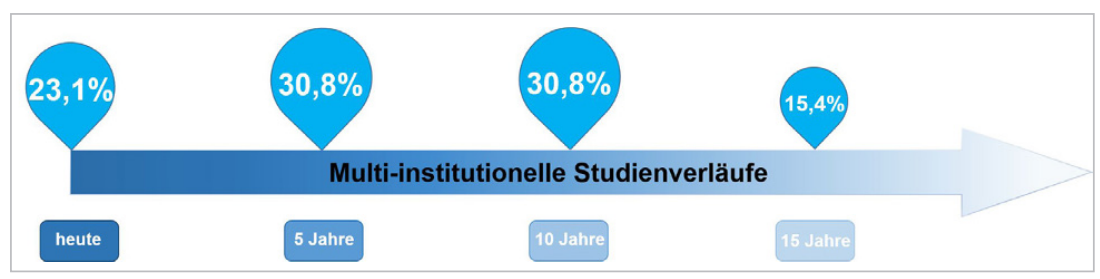

Abb. 51 Geschätzte Zeitdauer für die zunehmende Wichtigkeit multi-institutioneller Studienverläufe $(\mathrm{N}=39)$ 


\title{
C 3.1.3 Säule 3: Personalisierung akademischen Lernens
}

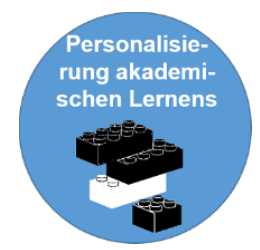

\begin{abstract}
Die Studiengangscurricula entwickeln sich von einer gänzlich vordefinierten und von vornherein gegebenen Struktur hin zu einem flexibleren, personalisierten und partizipativen Modell, in dem Studierende mit ihren Professorinnen und Professoren, Lehrenden, Ratgeberinnen und Ratgebern zur gemeinsamen Curricula-Entwicklung kooperieren.
\end{abstract}

Die Rolle der Studierenden wurde in Form eines dritten Faktors näher untersucht. Studierende würden künftig ihre eigenen, personalisierten Curricula in Zusammenarbeit mit den Lehrenden sowie Professorinnen und Professoren erstellen. Dies würde in der Konsequenz zu einer signifikanten Diversifizierung der Studiengangsoptionen führen. Zusätzlich hierzu würde eine Verschiebung in Richtung personalisierter Curricula zu einer Abkehr von der vordefinierten up-front-gegebenen Struktur führen, an deren Stelle ein partizipatives, personalisiertes Modell treten würde, in dem Studierende und Lehrpersonal miteinander kollaborierten, um gemeinsam Curricula für Studiengänge zu entwerfen. Die Zustimmung zu diesem Faktor war ähnlich hoch wie für die anderen Faktoren $\left(M=3,68, S D=0,98 ; A_{\text {Factor } 3}=54,4 \%\right)$. Die meisten der kritischen Kommentare, die sich als Hindernisse für die Entwicklung in Richtung personalisierter Curricula erweisen würden, sahen die Expertinnen und Experten in institutioneller Resistenz begründet. Als weiteren Aspekt nannte das Gremium der Expertinnen und Experten die Fähigkeiten der Studierenden, selbst tätig werden zu müssen, um den Umgang mit dieser gesteigerten Wahlfreiheit überhaupt bewältigen und davon profitieren zu können. Darüber hinaus ergaben sich Fragen bezüglich akademischer Qualitätskonzepte für diese Art der personalisierten künftigen Studienmodi.

In Bezug auf die Hochschulen sahen die Expertinnen und Experten einen hohen Bedarf für kulturellen Wandel, der sich entlang eines verstärkt bildungsorientierten Blickwinkels orientieren müsse. Obwohl die Sample-Mehrheit zustimmte, dass es für Studierende wünschenswert sei, sich ihre eigenen Curricula zu designen und mehr Wahlfreiheit zu haben, sprachen sich die Expertinnen und Experten aber auch für durch die Institutionen zu gewährleistende Sicherheitsnetze aus.

So geht der Grad an Personalisierung mit einem zu erhöhenden Angebot an Coaching und Unterstützung der Studierenden einher, um ihnen bei der Entwicklung ihrer eigenen akademischen Strukturen zu helfen und sie zu autonomen Lernenden zu entwickeln. Diese Funktion wurde als notwendig erachtet, um Lernende bei der Reflexion des Lernfortschritts zu unterstützen.

Natürlich bedarf es zur Umsetzung dieses Faktors eines radikalen Paradigmenwandels, mit dem Institutionen und Stakeholder der Hochschulen nicht vertraut 
sind. Allerdings scheinen heterogenere Zielgruppen und das Hinzukommen von nicht im traditionellen Sinn Studierenden mehr Raum für Personalisierungsmöglichkeiten notwendig zu machen, was sich in den hohen Zustimmungswerten der Expertinnen und Experten zeigt, während gleichzeitig Bedenken bezüglich der Implementierung solcher Personalisierungsmöglichkeiten aufkommen.

\section{Personalisierung akademischen Lernens: Geschätzte Zeitdauer für den Wandel}

Faktor drei kann laut der OECD mit Blick auf die steigenden Studierendenzahlen in industrialisierten Ländern als akuter Entwicklungstrend gesehen werden (Baethge et al. 2015; Teichler 2013; OECD 2016).

Dieser Trend hätte dann zur Folge, dass diversere Zielgruppen Hochschulbildungsangebote wahrnähmen, deren heterogenen Anforderungen Hochschulbildung nachkommen müsste. Personalisierung, Studieren in unterschiedlicher Geschwindigkeit und eine Vielzahl von Wahlmöglichkeiten für Studierende mit unterschiedlichen Hintergründen, die sich in unterschiedlichen Lebensphasen befinden, würden nach stärker personalisierbaren Ansätzen akademischer Bildung in Hochschulen verlangen. Es ist wahrscheinlich, dass der steigenden Zahl einer zunehmend heterogener werdenden Gruppe von Studierenden nur mit Hilfe von verbesserten Zielgruppen-orientierten Ansätzen begegnet werden kann. Diese Ansätze müssten in ihrer Struktur so angepasst werden, dass sie den heterogenen Lernbedürfnissen der Lernenden Rechnung tragen und auch sonst auf die künftig heterogener werdenden Bedürfnisse der Studierenden ausgerichtet sind.

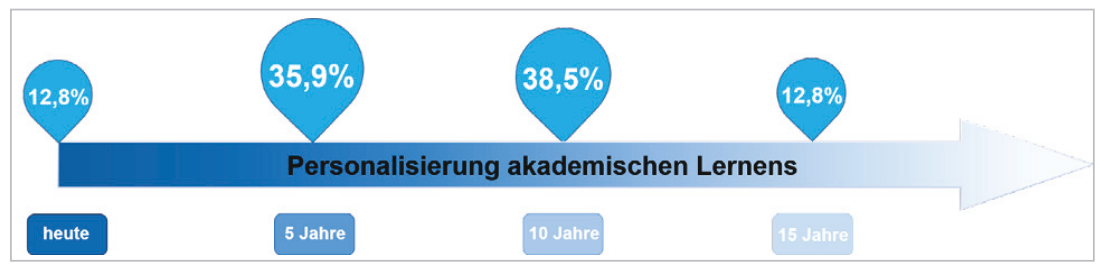

Abb. 52 Geschätzte Zeitdauer für die zunehmende Wichtigkeit personalisierten akademischen Lernens $(\mathrm{N}=39)$

Die Studiengangscurricula müssten sich in der Konsequenz von gänzlich vordefinierten und gegebenen Strukturen losmachen und sich flexiblerer, personalisierter und partizipatorischer Modelle bedienen, in denen Studierende aktiv mit 
Lehrenden, Professorinnen und Professoren, Beraterinnen und Beratern in der Curriculum- und Studiengangsgestaltung kooperieren. Personalisierung bedeutet dann auch, einzelne existierende Programme neu zusammenstellen zu können. Es gilt, die Studierenden bei den ersten Schritten in ihrer akademischen Laufbahn zu unterstützen, ihre Mission und ihre Leidenschaft zu entdecken. Und es bedeutet größere Wahlfreiheiten bezüglich der Lerninhalte und Module zuzulassen. In Summe läuft es also auf einen Gestalte dein eigenes Curriculum-Ansatz hinaus. Die Sample-Mehrheit gab an, dass dieser Faktor erst in den nächsten fünf (35,9\%) bis zehn Jahren (38,5\%) Realität werden würde (siehe Abbildung 52).

\section{3.1.4 Säule 4: Lebenslanges Lernen in Hochschulen}

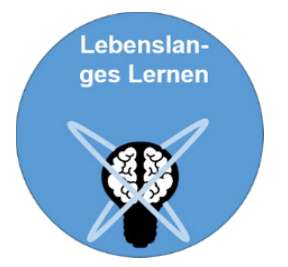

Das gegenwärtige Hochschulbildungsmodell sieht vor, Studierende auf ihren künftigen Beruf vorzubereiten. Dieses Modell wird durch Möglichkeiten für lebenslanges Lernens komplementiert.

Die vierte Säule des Wandels baut darauf, dass lebenslanges Lernen in Hochschulen ebenso wichtig wird wie der (momentane) Vorbereitungsmodus. Mit dem Begriff Vorbereitungsmodus wird das gegenwärtige Hochschulmodell adressiert. Dieses Modell basiert auf der Annahme, dass akademische Bildung dem Paradigma folgen sollte, bestimmte Wissensbestände zu erlernen, welche später auf einen professionellen Kontext angewendet werden und zur Aufgabenbearbeitung und -bewältigung im Berufsleben benötigt werden würde. Durch sich immer schneller wandelndes Wissen, Technologieakzeptanzraten und sich verändernde professionelle Kontexte ist das momentane Modell jedoch zunehmend erhöhtem Druck ausgesetzt. In dem Maße, in dem Hochschulen sich auf die Vermittlung von Wissen konzentrieren, jedoch in professionellen Handlungskontexten vor allem Future Skills benötigt werden, entsteht eine Kluft zwischen Anforderung und Angebot. Durch schnellere Innovationszyklen im Berufskontext zeichnet sich eine Verbreiterung dieser Kluft ab. Eine Möglichkeit dieses durch die Kluft entstehende Risiko abzumildern, besteht darin, den Fokus auf die Vermittlung von Future Skills zu verlagern, was auch das Vermitteln von Fachwissen einschließt, jedoch darüber hinaus geht.

Lebenslanges Lernen - oder für den Hochschulkontext lebenslanges akademisches Lernen - erlaubt es Studierenden und Arbeiternehmerinnen und Arbeitnehmern gleichermaßen, sich kontinuierlich weiterzubilden und an die neuen Herausforde- 
rungen ihres sich wandelnden Arbeitsfelds anpassen zu können. Dieses Konzept sollte und kann aber nicht nur aus der Perspektive eines Individuums gedacht werden. Vielmehr wird hierdurch die Notwendigkeit für einen Paradigmenwechsel der Hochschulbildung aufgezeigt: Akademische Bildung sollte nicht länger als Phase zu Beginn des Arbeitslebens gesehen, sondern als Kontinuum konstanter biographischer Lernepisoden verstanden werden.

Bachelor- und Masterqualifikationen müssen konsequenterweise dann als eine Grundphase am Anfang einer akademischen Laufbahn stehen, mit der ein Stadium nicht endet, sondern die nur die Fundamente für die weiteren Schritte legt, die ein immer wieder Einsteigen und Aussteigen in die akademischen Bildungsphasen bedeutet. Stanford nennt diese Phasen In-Loop und Out-Loop Zyklen. Transfer der erarbeiteten Leistungspunkte, Anerkennung bereits erbrachter Leistungen (akademischer und professioneller Natur), Kompatibilität unterschiedlicher Kompetenzen sowie flexible Verläufe würden es Individuen ermöglichen, sich ihre eigenen, individuellen lebenslangen Lernpfade selbst zu gestalten. Die Delphi-Befragten zeigten hohe Zustimmungswerte zur Entwicklung hin zum lebenslangen akademischen Lernen als gleichwertig zu dem derzeit vorherrschenden Vorbereitungsmodell $\left(M=3,72, S D=1,33 ; A_{\text {Factor4 }}=65,2 \%\right)$ (siehe Abbildung 53).

Obwohl die meisten der Expertinnen und Experten angaben, eine Fokusverschiebung in Richtung lebenslanges akademisches Lernen sei durchaus wünschenswert, wurden doch Zweifel darüber laut, ob Hochschulen die hierfür nötigen Veränderungen aus eigener Kraft würden leisten können. Sie wiesen auch auf die Notwendigkeit der Unterstützung durch die Hochschulgesetzgebung hin. Einige Expertinnen und Experten gaben zudem zu bedenken, dass lebenslanges höheres Lernen nicht notwendigerweise in der Hochschulbildung angesiedelt sein müsse, sondern als Individualverantwortung des Individuums gegenüber sich selbst verstanden werden müsse. Zuletzt könne nach Meinung der Befragten davon ausgegangen werden, dass externe Anbieter auch außerhalb der Hochschulen künftig eine wichtigere Rolle spielen würden.

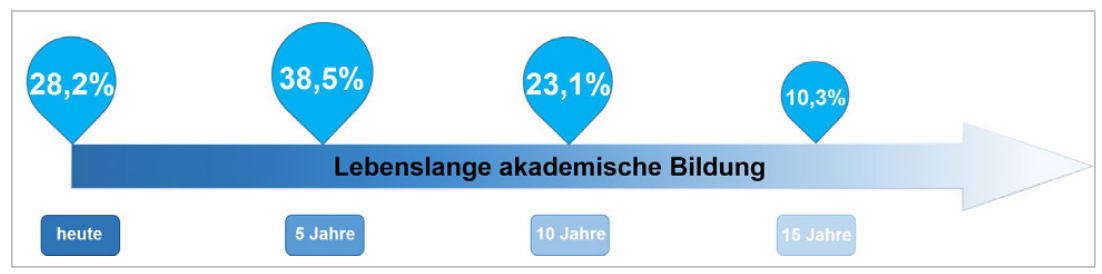

Abb. 53 Geschätzte Zeitdauer für die zunehmende Wichtigkeit lebenslanger akademischer Bildung $(\mathrm{N}=39)$ 


\section{Lebenslanges Lernen in Hochschulen: Geschätzte Zeitdauer für den Wandel}

Lebenslanges höheres Lernen erfreut sich - so die Meinung der Expertinnen und Experten - zunehmender Relevanz. Knapp ein Drittel (28,2\%) gab an, dass lebenslanges höheres Lernen bereits jetzt in einigen Hochschulen als ebenso wichtig wie die akademischen Standardabschlüsse angesehen würde. Vier von zehn Befragten glaubten dieser Trend würde erst in den nächsten fünf Jahren relevant werden $(38,5 \%)$ und etwa ein Viertel in den nächsten zehn Jahren. Etwa ein Viertel sprach sich dafür aus, dass dies erst in einem Zehnjahreszeitraum wichtig werden würde. Da es sich hierbei jedoch um einen systemischen Wandel handelt, erscheint ein Fünfjahreszeitraum sowohl in Hochschulen als auch für nötige Gesetzesveränderungen kurz. Allerdings gibt es auf nationaler sowie auf europäischer Ebene bereits durch Programme entwickelte Richtlinien, die den Fokus in Bildungssystemen sehr wohl innerhalb eines Fünf- bis Zehnjahreszeitraums auf lebenslanges höheres Lernen lenken könnten. Speziell unter den Bedingungen schnellen Wissenswandels, hohen Technologieakzeptanzraten und einem sich rasant wandelnden Arbeitskontext, könnte sich dieser Zeitrahmen sogar noch verkürzen.

\section{3.2 Vier Szenarien für die Hochschule der Zukunft}

Das Studium ist zum Normalfall geworden. Durch den digitalen Wandel entstehen völlig neue Lernwege. Das derzeit vorherrschende Modell eines drei- bis fünfjährigen Studienblocks bei nachfolgender lebenslanger Arbeitstätigkeit verliert an Relevanz. An seine Stelle treten flexiblere, oft lebenslange Studienmodelle. Hochschulen, Politik und Gesellschaft müssen hierfür zusammen mit den Studierenden die Voraussetzungen schaffen und einen neuen Leitkonsens für die Hochschule der Zukunft erarbeiten. Auch die Erwartungen und Anforderungen der Studierenden ändern sich schnell. Schon heute sind sie als Gruppe deutlich vielfältiger als es zuvor der Fall war (siehe Kapitel C 1.3 Dritte Sekunde: Der demographische Wandel). So verfügte im Jahr 2016 mehr als jeder fünfte Studierende über eine abgeschlossene Berufsausbildung, jeder zehnte studierte trotz einer gesundheitlichen Beeinträchtigung und jeder fünfzigste Studierende hatte kein Abitur.

Wie die Hochschule der Zukunft aussehen wird, hängt sicher von den jeweils regional-lokalen Kontextbedingungen ab, in denen sie sich entwickeln wird. Sicher ist, die Hochschule der Zukunft wird anders aussehen als die gegenwärtige. Die Einflussfaktoren (siehe Kapitel C1) sind zu mächtig und zu vielfältig. Leitet man $a b$, welche Haupteinflussfaktoren sich daraus ergeben, kommt man zu den vier 
Säulen des Wandels, die im vorherigen Kapitel beschrieben wurden. Sie eröffnen einen Raum für künftige Entwicklungen in der Hochschulbildung. Die Studienerfahrung wird sich in Abhängigkeit der jeweiligen Konfiguration und der Intensität der Entwicklungen der vier Säulen verändern.

Im Rahmen der NextSkills Studie wurden Expertinnen und Experten vier verschiedene Szenarien vorgelegt, die auf der Annahme basieren, die vier dargestellten Faktoren (siehe Kapitel C 3.1 Entwicklungsrahmen für die Hochschule der Zukunft: Vier Säulen für den Hochschulwandel) würden effektiv werden. Tabelle 4 zeigt die Bedingungen der vier Szenarien auf. Diese bauen auf einem Basisszenario auf, in welchem alle vier Säulen in geringer Intensität bereits existieren. Das abgeleitete Basisszenario für die heutige Studienerfahrung stellt sich wie folgt dar:

\section{Basisszenario heute: Business as usual}

Hochschulbildung ist generell institutionsgebunden - Studierende schreiben sich - für gewöhnlich direkt nach ihrem Schulabschluss an einer Institution ein, an der sie später auch ihren Abschluss machen. Sie studieren entlang eines vordefinierten Curriculums mit vorgegebenen Inhalten, um vorgeschriebene Lernziele zu erreichen, die sich in der Regel aus einem bestimmten Berufsfeld oder einer Wissenschafts disziplin ableiten. Studiengänge sind dabei in einer akademischen Disziplin oder Fakultät/ Organisationseinheit des gegenwärtigen akademischen Systems verortet.

Basierend auf dem Basiszenario in welchem die vier Faktoren nur in geringer Intensität auftreten, wurden vier Szenarien erstellt. Ihnen liegt eine kumulative Logik zugrunde, jeweils im nächsten Schritt wird dem vorherigen Szenario ein weiterer Faktor hinzugefügt. Tabelle 4 zeigt die unterschiedliche Konfiguration der vier Faktoren in den Szenarien - jeweils formuliert aus Studierendenperspektive. Wie aus der Tabelle ersichtlich, bauen die Szenarien aufeinander auf. Die Delphi-Befragten wurden gebeten, den Grad ihrer Zustimmung zu den vorgeschlagenen Szenarien auf einer fünf-stufigen Likert-Skala anzugeben, die von starker Zustimmung (=5) bis hin zu starker Ablehnung (=1) reichte (siehe Abbildung 54). Zusätzlich erhielten die Befragten die Möglichkeit, eine Begründung für Ihre Entscheidung oder Anmerkungen für potentielle Reformulierungen der Szenarienbeschreibung in einem Freitextfeld anzugeben. 
Tab. 4 Methode der Szenarioformierung für die momentane und künftige Hochschulbildung aus Studierendenperspektive

\begin{tabular}{|c|c|c|c|c|c|}
\hline $\begin{array}{l}\text { Einfluss- } \\
\text { Faktoren }\end{array}$ & $\begin{array}{l}\text { Szenario: } \\
\text { Heute }\end{array}$ & $\begin{array}{l}\text { Szenario: } \\
\text { Zukunft } 1\end{array}$ & $\begin{array}{l}\text { Szenario: } \\
\text { Zukunft } 2\end{array}$ & $\begin{array}{l}\text { Szenario: } \\
\text { Zukunft } 3\end{array}$ & $\begin{array}{l}\text { Szenario: } \\
\text { Zukunft } 4\end{array}$ \\
\hline $\begin{array}{l}\text { Future } \\
\text { Skills }\end{array}$ & $\begin{array}{l}\text { Geringe } \\
\text { Intensität }\end{array}$ & $\begin{array}{l}\text { Hohe } \\
\text { Intensität }\end{array}$ & $\begin{array}{l}\text { Hohe } \\
\text { Intensität }\end{array}$ & $\begin{array}{l}\text { Hohe } \\
\text { Intensität }\end{array}$ & $\begin{array}{l}\text { Hohe } \\
\text { Intensität }\end{array}$ \\
\hline $\begin{array}{l}\text { Multi- } \\
\text { institutionell }\end{array}$ & $\begin{array}{l}\text { Geringe } \\
\text { Intensität }\end{array}$ & $\begin{array}{l}\text { Geringe } \\
\text { Intensität }\end{array}$ & $\begin{array}{l}\text { Hohe } \\
\text { Intensität }\end{array}$ & $\begin{array}{l}\text { Hohe } \\
\text { Intensität }\end{array}$ & $\begin{array}{l}\text { Hohe } \\
\text { Intensität }\end{array}$ \\
\hline $\begin{array}{l}\text { Personali- } \\
\text { sierung }\end{array}$ & $\begin{array}{l}\text { Geringe } \\
\text { Intensität }\end{array}$ & $\begin{array}{l}\text { Geringe } \\
\text { Intensität }\end{array}$ & $\begin{array}{l}\text { Geringe } \\
\text { Intensität }\end{array}$ & $\begin{array}{l}\text { Hohe } \\
\text { Intensität }\end{array}$ & $\begin{array}{l}\text { Hohe } \\
\text { Intensität }\end{array}$ \\
\hline $\begin{array}{l}\text { Lebenslanges } \\
\text { lernen }\end{array}$ & $\begin{array}{l}\text { Geringe } \\
\text { Intensität }\end{array}$ & $\begin{array}{l}\text { Geringe } \\
\text { Intensität }\end{array}$ & $\begin{array}{l}\text { Geringe } \\
\text { Intensität }\end{array}$ & $\begin{array}{l}\text { Geringe } \\
\text { Intensität }\end{array}$ & $\begin{array}{l}\text { Hohe } \\
\text { Intensität }\end{array}$ \\
\hline
\end{tabular}

In der folgenden Infografik werden die vier Szenarien zusammenfassend dargestellt und die Zustimmungswerte zu den Szenarien abgebildet (Abbildung 55). 


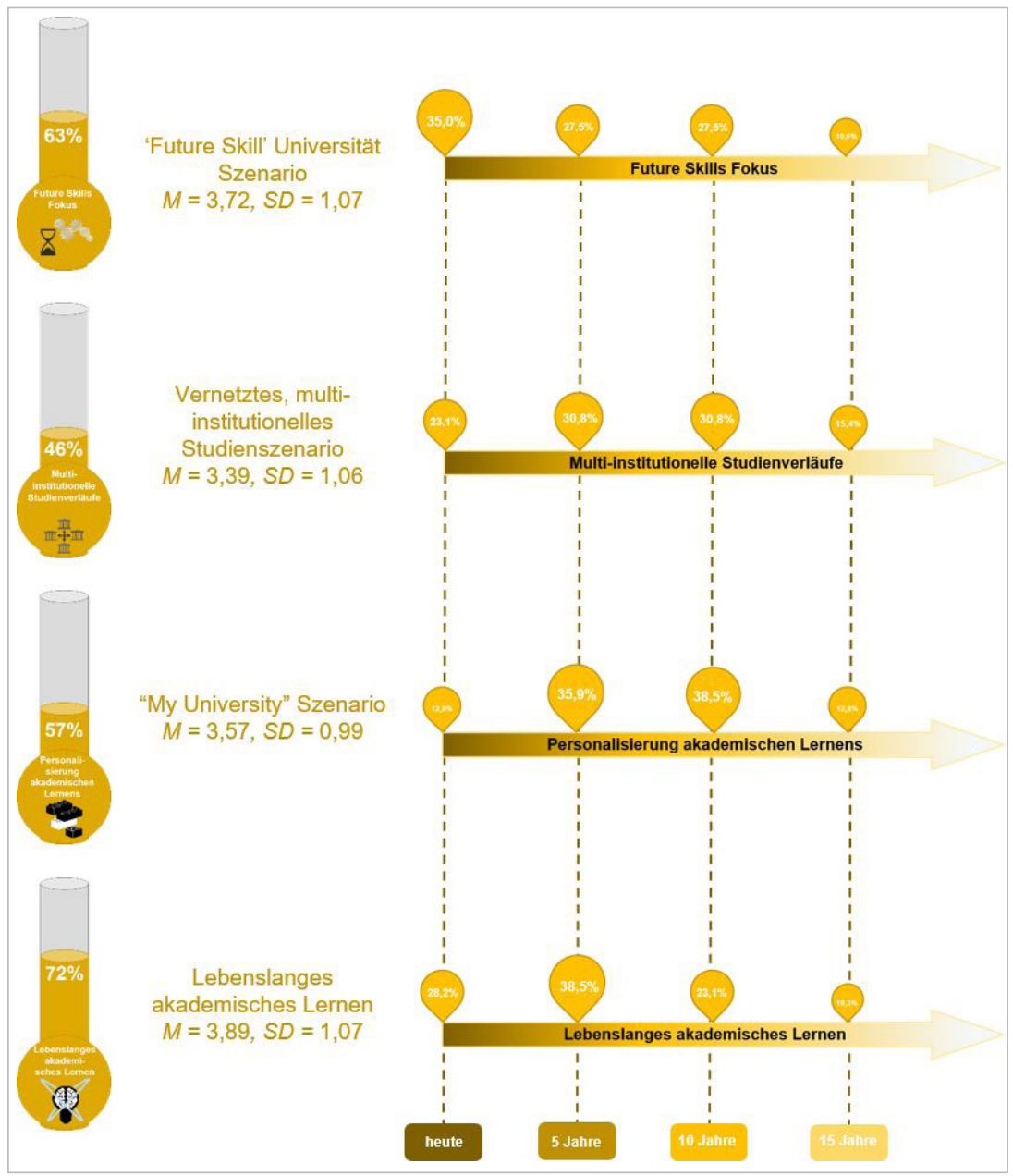

Abb. 54 Überblick über die Zustimmungslevel zu und geschätzte Zeitdauer potentieller Szenarien für Hochschulbildung aus Studierendenperspektive $\left(\mathrm{N}_{\text {Rundel }}=46\right.$, $\mathrm{N}_{\text {Runde2 }}=38$ ) 

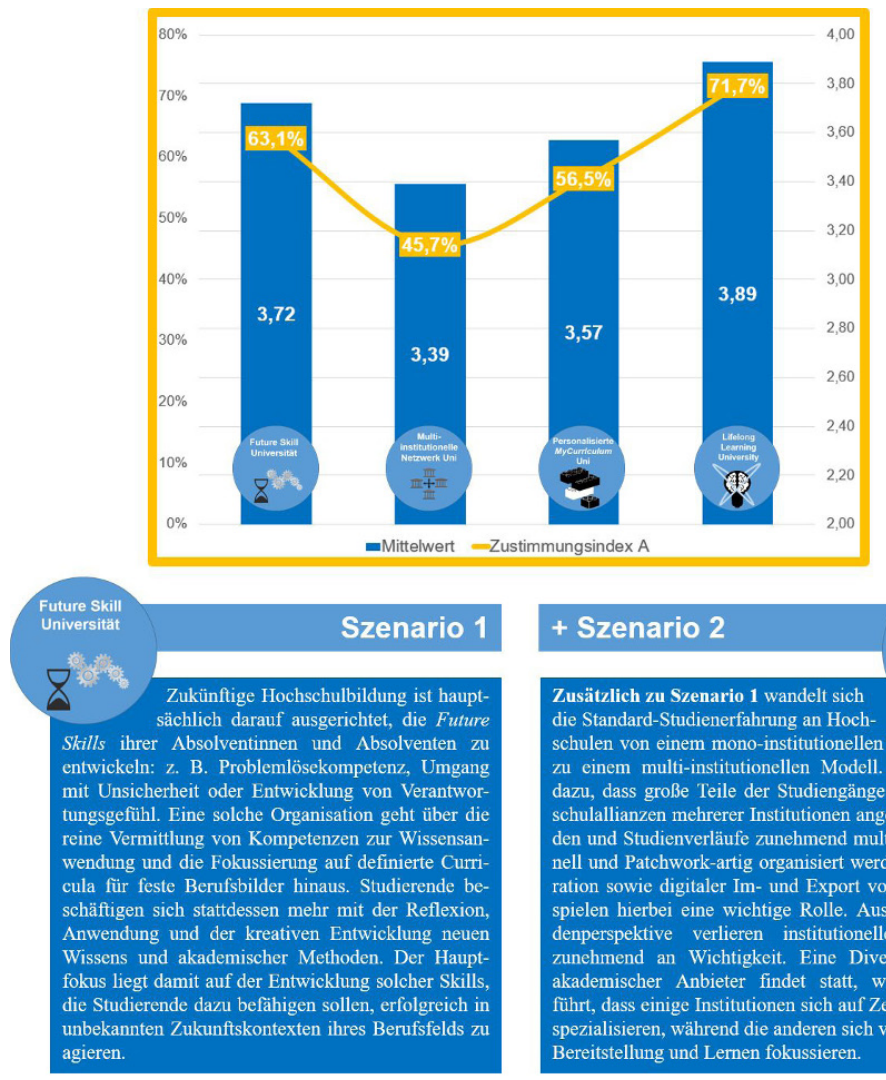

Zusätzlich zu Szenario 1 wandelt sich

die Standard-Studienerfahrung an Hoch-

schulen von einem mono-institutionellen Modell hin zu einem multi-institutionellen Modell. Dies führt dazu, dass große Teile der Studiengänge von Hochschulallianzen mehrerer Institutionen angeboten werden und Studienverläufe zunehmend multi-institutionell und Patchwork-artig organisiert werden. Kooperation sowie digitaler Im- und Export von Curricula spielen hierbei eine wichtige Rolle. Aus Studierendenperspektive verlieren institutionelle Grenzen zunehmend an Wichtigkeit. Eine Diversifizierung akademischer Anbieter findet statt, welche dazu führt, dass einige Institutionen sich auf Zertifizierung spezialisieren, während die anderen sich verstärkt auf Bereitstellung und Lernen fokussieren.

Zusätzlich zu Szenario 2 erhöht sich der studentische Grad der Autonomie erheblich. Studierende werden von Professorinnen und Professoren, Lehrenden, Beraterinnen und Beratern angeleitet, um sie zu autonomen Lernenden zu entwickeln, die sich selbst mit der Zusammenstellung ihres Curriculums befassen, indem sie ihr akademisches Studienportfolio aus verschiedenen Studiengängen und aus dem Angebot unterschiedlicher Institutionen zusammenstellen. Fachdisziplingrenzen von Studiengängen werden flexibler und ermöglichen Studierenden so individuelle Studienverläufe. Der Hauptfokus liegt damit auf dem wachsenden Grad an Autonomie der Studierenden und ihren persönlichen Studienbedürfnissen sowie dem zukünftigen professioPersonalisierte
MyCurriculum
nellen Kontext. MyCurricu
Uni

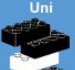

+ Szenario 3

Zusätzlich zu Szenario 3 wird nahtloses lebenslanges höheres Lemen ebenso wichtig wie die initiale Hochschulbildung. Berufstätige machen den Großteil der Studierenden aus. Studierende wählen ihr ModulPortfolio entsprechend ihrer persönlichen SkillBedürfnisse und Kompetenz-Bedarfe mit einem hohen $\mathrm{Maß}$ an Autonomie über ihr Leben hinweg aus. Institutionen bieten Micro-Credentials an, welche die Studierenden individuell und ihren eigenen Interessen und Bedürfnissen entsprechend erwerben können.

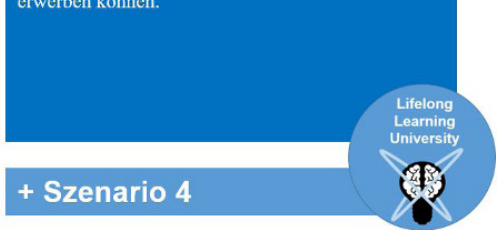

Abb. 55 Vier Szenarien für die Hochschule der Zukunft im Überblick 


\section{3.2.1 Szenario 1: Die Future Skill Universität}

Szenario Nummer 1, das Future Skills Szenario, geht davon aus, dass Hochschulen sich von ihrem momentanen Modell der Wissensvermittlung abkehren würden. Anstelle dessen würden neue Studiengangs- und Hochschulprofile entstehen, die auf die Entwicklung von Future Skills abzielen. In diesem Szenario wäre Hochschulbildung auf ein Hauptziel ausgerichtet: die Entwicklung von Future Skills zu ermöglichen, das heißt Handlungsfähigkeit in hochemergenten Kontexten eines möglichen zukünftigen Professionsfeldes. Dies würden Wissensaneignung und definierte Curricula für feststehende Berufsbilder zwar nicht ersetzen, aber doch weit über sie hinausgehen. So würde der Fokus der Studierenden stattdessen auf Reflexion über Werte und Einstellungen, Anwendung sowie der kreativen Entwicklung neuen Wissens und akademischer Methoden liegen. Die Hauptambition innerhalb dieses Szenarios wäre damit, Studierende darauf vorzubereiten, in ungewisseren und unsicheren Zukunftskontexten handlungsfähig zu sein.

Die Delphi-Expertinnen und -Experten drückten hohe Zustimmung zu diesem Szenario aus $\left(M=3,68, S D=1,07, A_{\text {scenariol }}=63,1 \%\right)$. Future Skills werden dabei verstanden als ein Konzept, das auf Fachwissen aufbaut, aber darüber hinaus geht, um Studierenden das Erschließen höherer Lernebenen (beispielsweise auf der Taxonomie von Bloom) zu ermöglichen, die für die künftige akademische Bildung notwendig sein werden. Die Expertinnen und Experten stimmte zu, dass Hochschulen Lernmöglichkeiten anbieten müssten, welche auf die Entwicklung von Future Skills abzielten. Zudem wurde jedoch angemerkt, dass die Definition und der Umfang davon, was als Future Skills betrachtet würde, von der jeweiligen Disziplin abhängig sei. 


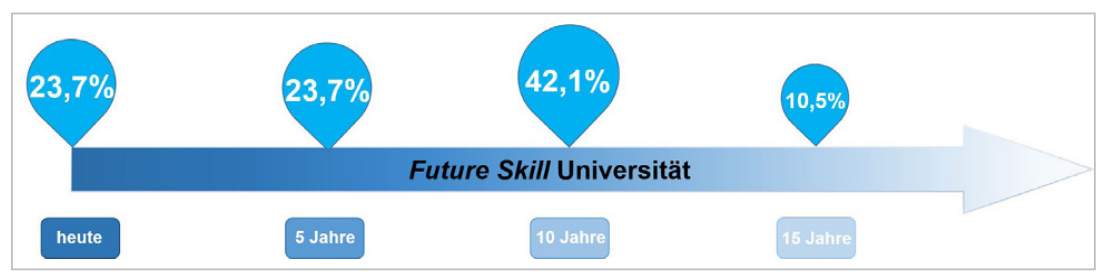

Abb. 56 Geschätzte Zeitdauer für die zunehmende Wichtigkeit des Future Skill Universität Szenarios $(\mathrm{N}=46)$

Die Mehrheit der Befragten (42,1\%) schätzt den Zeitraum für die Realisierung dieses Szenarios auf zehn Jahre ein $(M=2,39, S D=0,97)$ (siehe Abbildung 56). Die Analyse der qualitativen Kommentare ergab, dass der Hauptgrund für die Schätzung der Expertinnen und Experten einer nur mittelfristigen Einführung dieses Szenarios vor allem in vier Faktoren begründet sei: in der Trägheit der Hochschulen, deren internem kulturellen Widerstand sowie nur langsam ablaufenden Veränderungsprozessen in Bezug auf Gesetzesänderungen und Finanzierungsregeln. Zudem wurde betont, dass neben den Makro- und Meso-Level Veränderungen (Landespolitik sowie institutionelle Verordnungen), auch das Hochschulpersonal und die Studierenden ihr Mindset entsprechend anpassen müssten.

Als Moderatoren solcher Wandlungsprozesse wurden Heterogenität der jeweiligen nationalen Kontextbedingungen und der verschiedenen Hochschultypen identifiziert. Während einige Befragte angaben, dass sich private Hochschulen und Business Schools bereits aktiv darum bemühten, ihre Studierenden bei der Entwicklung von Future Skills zu unterstützen, würden oft vor allem traditionelle Universitäten hauptsächlich Wert auf Wissensvermittlung legen.

\footnotetext{
"It is already a reality that the knowledge needed for a certain profession is evolving constantly and needs a permanent update. So, having the skills for this adaptation and having skills more focused on developing capacities is much more important than just information and knowledge in a specific field." (Teilnehmer an NextSkills Delphi-Studie)
} 


\section{3.2.2 Szenario 2: Die multi-institutionelle Netzwerk Universität}

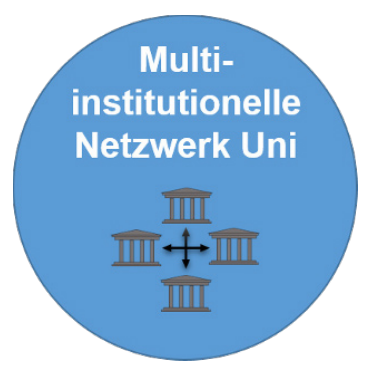

Das zweite Szenario geht davon aus, dass sich zusätzlich zum Fokus auf Future Skills in Hochschulen eine Hochschulstruktur etabliert, die neue Studienerfahrungen in einem Netzwerk vieler Hochschulinstitutionen ermöglicht, hier mit Multi-Institutionalität bezeichnet. Dadurch wird der Dreh- und Angelpunkt bisheriger Hochschulbildung verschoben. Dreht sich bislang alles um die Achse der einzelnen Hochschule, an der sich von der Einschreibung bis zur Graduierung alles vollzieht, so wird diese Achse nun komplett gedreht. Das Szenario sieht ein Studium vor, welches sich nicht mehr um die Achse der Institution als Mittelpunkt dreht, sondern um das Studium. Dabei steht die Frage im Mittelpunkt, wie durch institutionelle Kooperation ganz neue Studienerfahrungen ermöglicht werden können. Der Dreh- und Angelpunkt für die Definition eines Hochschulstudiums wird gänzlich verschoben.

Das erfordert ein radikales Umdenken des bisherigen Hochschulkonzeptes. Dieses basiert auf der Annahme, dass Hochschulen einerseits ein Studium aus einer Hand ermöglichen, gewissermaßen als Full-Service-Anbieter, und zweitens, dass sie auch Räume für personelle und organisationale Identitätsentwicklung darstellen, die als Campus Mentalität oder mit Begriffen wie alma mater belegt sind. Im neuen Konzept der multiinstitutionellen Netzwerk-Hochschule geht es darum, die Vorteile der Vernetzung zu ermöglichen während gleichzeitig der identitätsbildende Raum des Ein-Hochschulmodells - auch im Sinne eines Schutzraumes - nicht aufgegeben werden soll. Hierfür muss die Idee der bisherigen Hochschulorganisation neu gedacht werden. Wie kann ein Studium an verschiedenen Institutionen in einer Netzwerkstruktur trotzdem so gestaltet werden, dass die vermutete Kohärenz des Ein-Hochschulmodells nicht verloren geht.

Bisherige Ansätze, insbesondere in den USA, erscheinen oft eher dem neoliberalen Marktmodell verschrieben und versuchen über Skaleneffekte Kostenvorteile herauszuarbeiten (vgl. Selingo 2017). Auch in Europa sind Ansätze der vernetzten Universität in unterschiedlichen Gestalten in Diskussion, beispielsweise in Form 
der sogenannten „European University“ Initiative der Europäischen Kommission. Hierbei sollen sich Netzwerke von Hochschulinstitutionen in Europa zusammenfinden, die Kooperation in Studium und Forschung neu denken. ${ }^{50}$ Auch regionale Netzwerke etablieren sich mehr und mehr, bislang jedoch zumeist vor allem mit Zielen im Bereich der Forschung. Ein Beispiel ist das „Netzwerk mittelgroßer Universitäten" in Deutschland, ein Netzwerk primär für die Forschungskooperation. ${ }^{51}$ Das stärkste Innovationspotenzial liegt jedoch in der Möglichkeit, gänzlich neue Studiererfahrungen zu ermöglichen. Das Szenario der multi-institutionellen Netzwerk Universität bezieht sich auf diesen Blickwinkel.

Das Szenario einer multi-institutionellen Netzwerk Universität folgt dem Gedanken radikaler Integration der Curricula verschiedener Hochschulen in einem Netzwerk. Für Hochschulen bedeutet dies verstärkt Im- und Export von curricularen Anteilen anderer Hochschulinstitutionen. Diese Integration von Studienangeboten durch Digitalisierung wird zukünftig stark an Bedeutung zunehmen und einfacher werden. Die zunehmende Verfügbarkeit von offenen Onlinekursen und offenen Bildungsmaterialien spricht dafür, dass Studiererfahrungen zunehmend weniger institutionengebunden bleiben müssen. Jedoch wäre es ein Irrglaube zu denken, dass Digitalisierung automatisch zu (sinnvoll) vernetzten Studienangeboten führt. Hierfür gilt es, Werkzeuge der Anerkennung auszuarbeiten und bereitzustellen. Studienerfahrungen sind dabei nicht mehr von Hochschulen verwaltete Leistungspunkte, die nur in bestimmten, durch Hochschulen selber regulierten Kombinationen zu einem Studienabschluss führen, sondern werden zu portablen Studienleistungen, die in der persönlichen Verantwortung Studierender liegen. Das hierfür notwendige Konzept des persönlich verantworteten und durch Hochschulen ermöglichten sowie weiterhin durch Lehrende und Coaches beratenen akademischen Qualifikationskonzeptes ist derzeit noch nicht erprobt oder konzipiert. Im NextSkills Delphi weisen die Befragten darauf hin, dass es sich einerseits abzeichnet, dass diese Entwicklung bevorsteht, andererseits auch Risiken im Hinblick auf die Kohärenz und Anschlussfähigkeit der Studienerfahrungen bestehen.

Die Standard-Studienerfahrung an Hochschulen wandelt sich in diesem Szenario von einem ein-institutionellen Modell hin zu einem multi-institutionellen Modell (siehe Abbildung 58). Für Studierende bedeutet dies, dass sie sich zwar an Hochschule 1 einschreiben, deshalb aber nicht notwendigerweise auch an dieser Hochschule graduieren oder

50 Siehe hierzu die Beschreibung der Initiative auf den Internetseiten der europäischen Kommission: https://ec.europa.eu/education/education-in-the-eu/european-education-area/european-universities-initiative_en

51 Siehe dazu die Website des Netzwerks: http://www.mittelgrosse-universitaeten.de 

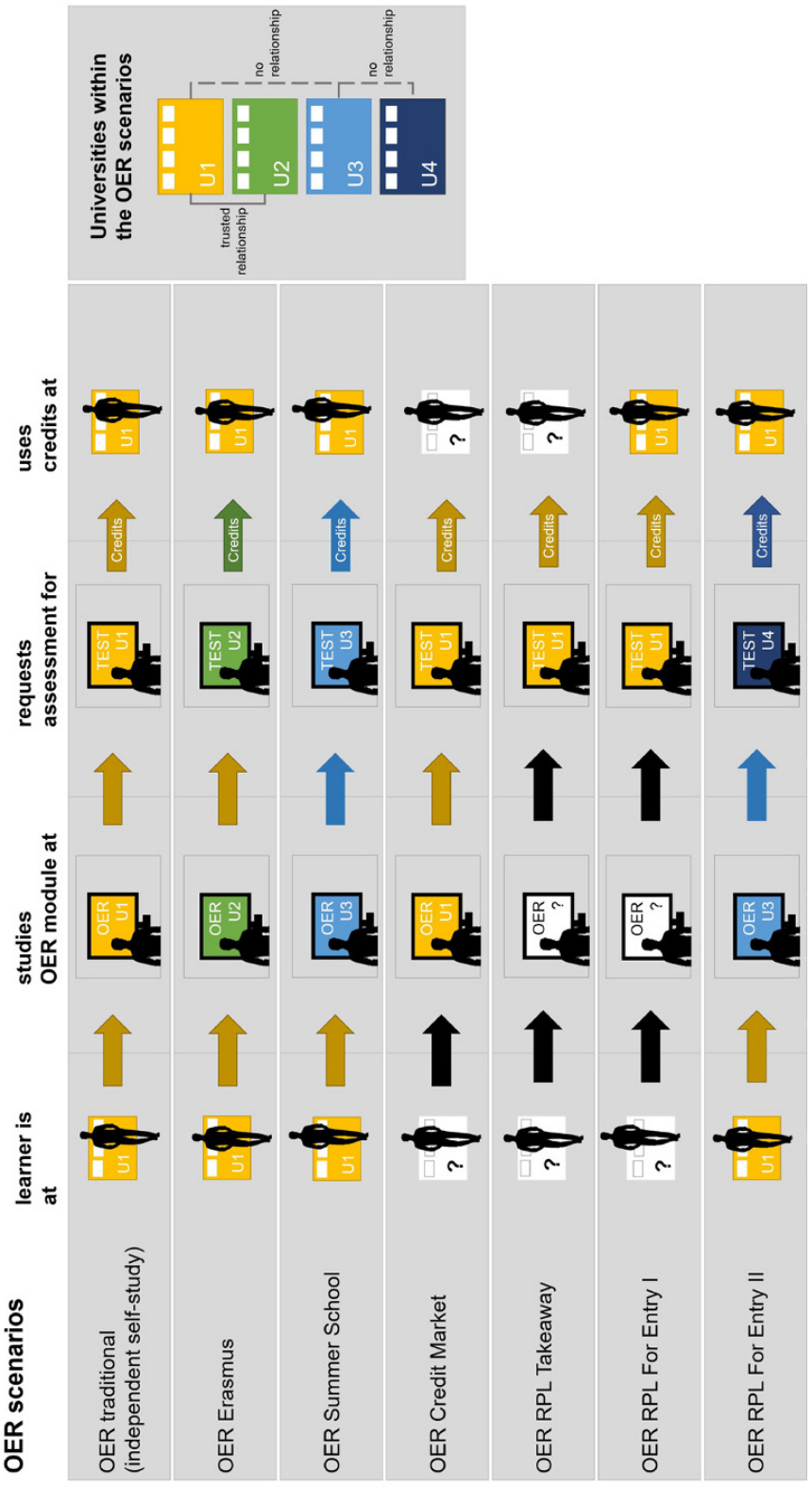

Abb. 57 Szenarien multi-Institutioneller Studierpfade (Quelle: nach Ehlers et al. 2011) 
alle Kurse nur an dieser Hochschule belegen. Stattdessen wird das Studium zu einer Patchwork-, beziehungsweise Netzwerk-Erfahrung, die mehr von den jeweiligen Interessen und individuellen Präferenzen der Studierenden für bestimmte Themen und Profile, gewünschtem Zugang zu spezifischen Einrichtungen oder Kursprofilen abhängt als von den curricularen Möglichkeiten und Kapazitäten der Hochschule oder deren Kompetenzen, ein bestimmtes Studienangebot anzubieten. Sollten diese nicht gegeben sein, so nehmen Studierende an einer anderen Institution entsprechende Angebote wahr. Aus Perspektive der Institutionen würde dies bedeuten, dass sie sich in Allianzen und Netzwerken mit anderen Hochschulen und Hochschulbildungs-Anbietern organisieren müssten, Kooperationsschnittstellen sowie gegenseitig anerkennungsfähige, portable Leistungspunkteschemata und Zertifikate entwickeln müssten. Akademische Programme würden in diesem Szenario entsprechend durch Hochschul-Allianzen bereitgestellt und würden es Studierenden ermöglichen, individuelle, patchworkartige Studienverläufe zusammenzustellen, die institutionelle Grenzen überschreiten. Dies würde durch Kooperation sowie durch den digitalen Im- und Export von Curricula ermöglicht werden. Aus Studierendenperspektive würden institutionelle Grenzen daher weniger sichtbar und es würde sich eine Diversifikation der Anbieter akademischer Bildung ergeben, in deren Konsequenz sich einige Institutionen auf Zertifizierung spezialisierten, einige auf Coaching und Lernbegleitung, während die anderen sich verstärkt auf Bereitstellung von Inhalten fokussierten. Denkbare multi-institutionelle Studierpfade, die so ermöglicht würden, werden in Abbildung 57 dargestellt.

Die Teilnehmerinnen und Teilnehmer des NextSkills Delphi wurden gebeten, das Szenario hinsichtlich ihrer Zustimmung und hinsichtlich der Zeitspanne der Realisierung einzuschätzen. Es zeigt sich einerseits eine vorsichtige Zustimmung zum Szenario mit einem Mittelwert von 3,43 $(S D=1,06)$ und einem Zustimmungsindex A von 45,7\% (siehe Abbildung 58). ${ }^{52}$ Allerdings schätzen die Befragten die time of adoption für dieses Szenario als langsamer ein, mit einem Schwerpunkt der Befragten (36,8\%) die hierfür einen Zehnjahreszeitraum vermuten und $21,1 \%$, die diese Entwicklung erst in einem Fünfzehnjahreszeitraum sehen. Mehr als die Hälfte der Befragten schätzen dieses Szenario also eher in einer langfristigen Perspektive als realistisch ein.

52 Der Zustimmungswert A (für Agreement index) gibt den prozentualen Anteil derjenigen an, die mit 4 oder 5 (Zustimmung bzw. starke Zustimmung) auf der 5-stufigen Likert-Skala geantwortet haben. 


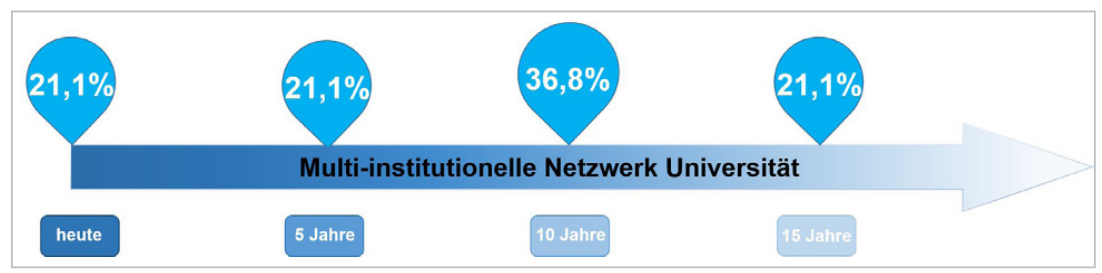

Abb. 58 Geschätzte Zeitdauer für die zunehmende Wichtigkeit des vernetzten, multiinstitutionellen Studienszenarios $(\mathrm{N}=46)$

Expertinnen und Experten gaben an, dass neue Geschäftsmodelle entlang einer „Coopetition“ entwickelt werden müssten. ${ }^{53}$ Sie gaben ebenfalls an, dass Hochschulen die Bereitschaft zu neuen Wettbewerbsmodellen akzeptieren müssten, um dieses Szenario möglich zu machen. Konstruktive Diskussionen wären daher nötig, um die Konditionen näher zu spezifizieren unter welchen Hochschulen sich in Richtung kooperativer Netzwerke entwickeln könnten ohne dabei die Bedingungen aus den Augen zu verlieren, die zu einer Schwächung der eigenen Wettbewerbsposition führen würden. Geschäftsmodelle, die Instrumente zur Anerkennung und zum Punktetransfer beinhalten, müssten ebenfalls entwickelt werden, um die Entstehung solcher institutionen-umspannenden Arrangements stärker zu motivieren. Nach Meinung von Expertinnen und Experten wird die studentische Nachfrage dieses Szenario vorantreiben:

\begin{abstract}
"A rather neo-liberal ideal of a market place with infinite choice and competition but at what cost? I'm not sure this is as liberating as it sounds." (Teilnehmer an NextSkills Delphi-Studie)
\end{abstract}

Das zweite Szenario wird - so die Einschätzung der Expertinnen und Experten innerhalb der nächsten zehn bis fünfzehn Jahre realistisch zur Umsetzung kommen. Allerdings benennen die internationalen Expertinnen und Experten bestimmte Voraussetzungen für die Realisierung dieses Szenarios:

- Der Wille der Hochschulen ihre eigene Identität und ihren Status zu bewahren, während sie sich in einen intensiven Kooperations- und Anerkennungsprozess

53 Coopetition bzw. Kooperationswettbewerb, auch Koopkurrenz, bezeichnet die Dualität von Konkurrenz und Kooperation auf Märkten (Bouncken et al. 2015). Coopetition ist ein aus den englischen Begriffen cooperation (Kooperation) und competition (Wettbewerb) zusammengesetztes Kofferwort. 
begeben, könnte als Verlustrisiko für die Verortung von Studiengängen an der eigenen Hochschule angesehen werden.

- Obwohl einige der Befragten die Vorteile dieses Szenarios betonten, stellten andere in Frage, ob multi-institutionelle länder- und hochschulartenübergreifende Arrangements überhaupt anwendbar und wünschenswert seien.

- Das Konzept der Übertragbarkeit von Studienleistungen müsse zunächst reifen, bevor Hochschulen davon in beide Richtungen Gebrauch machen könnten: einerseits als selbst ausstellende, andererseits als anerkennende Institutionen.

Im Szenario der multi-institutionellen Netzwerk Universität spielt die Begleitung der Studierenden bei institutionen- und hochschulkontextübergreifender Studienerfahrung eine wichtige Rolle. Das Unterstützen von Studierenden bei der Entwicklung des roten Fadens in ihrem Studium wird in dem Maße bedeutender, in dem im äußeren Rahmen weniger Vorgaben existieren. In der Erarbeitung dieses Szenarios wird ebenfalls deutlich, dass die Infrastruktur für Coaching, Mentoring und die Begleitung von Studierenden, ihre akademischen Lernerfahrungen zu einem großen Ganzen zu integrieren auch stark ausgebaut werden müsste. Ansonsten bestünde ein Risiko des Verlusts an Kohärenz.

\section{3.2.3 Szenario 3: \\ Die personalisierte MyCurriculum Universität}

Das MyCurriculum Szenario fokussiert auf den Ausbau der Beteiligung von Studierenden an ihren Curricula. Mehr Wahlfächer, mehr Mitbestimmung und mehr Möglichkeiten, tatsächlich den eigenen Studienverlauf zusammenzustellen. Natürlich nicht allein, sondern in Zusammenarbeit mit Professorinnen und Professoren, Coaches oder entsprechenden Gremien. Studierende können dabei ihre eigenen Curricula und Lernformen basierend auf ihren persönlichen Interessen zusammenstellen. Das Curriculumkonzept für akademische Programme verändert sich also fundamental in diesem Szenario - weg von einer up-front gegebenen Struktur hin zu 
einem personalisierten, flexibleren und partizipativen Modell, in dem Studierende aktiv mit Professorinnen und Professoren, Lehrenden, Beraterinnen und Beratern kooperieren, um ihr eigenes Curriculum zu entwickeln. Zur Eigendefinitionshoheit in Bezug auf Curricula kommt zusätzlich eine größere Wahlfreiheit und Diversität in Bezug auf Lehrformate, durch die Hochschulen besser als bislang in der Lage sein werden, auf die zukünftig größere Diversität der Studierenden einzugehen (siehe Kapitel C 1.3 Dritte Sekunde: Der demographische Wandel). Diese kann sich entlang der Unterstützungsbedarfe, der mehr oder weniger vertieften Interessen zu bestimmten Studieninhalten, den unterschiedlichen Studiengeschwindigkeiten, den unterschiedlichen Präferenzen bezüglich Online- und Präsenzstudium oder auch in Bezug auf Internationalität widerspiegeln.

In der Konsequenz wird die starre Gebundenheit der überwiegenden Mehrheit der akademischen Programme an eine akademische Organisationseinheit (Fakultät, School, etc.) aufgelöst. Der Hauptfokus liegt damit auf den individuellen Studienbedürfnissen der Studierenden sowie deren zukünftigen professionellen Kontexten in einem personalisierten Studium. Die Mehrheit der Expertinnen und Experten betonte, dass dies nicht gleichzusetzen sei mit einer Auflösung von akademisch-wissenschaftlichen Fachdisziplinen. Sondern sich stattdessen vielmehr disziplinübergreifende Kooperationen für akademische Hochschulstudiengänge ergeben würden, die flexiblere, personalisierte Curricula ermöglichten. Erste Ansätze dazu zeigen sich bereits gerade in privaten Hochschulen, so etwa in der Jacobs Universität, einer privaten Hochschule aus dem Norden Deutschlands. Ein Experte, der selbst einen stark personalisierten Studiengang durchlief, schätzt seine eigene Erfahrung folgendermaßen ein:

\footnotetext{
“The challenge was creating a balanced degree' (temptation to choose 'easy' or familiar subjects) so some criteria should be agreed on, such as a balance between practical/ theoretical, subject-related/soft skills. Future professional contexts are uncertain, so this scenario needs to be associated with sound orientation approaches, both human and machine-based." (Teilnehmer an NextSkills Delphi-Studie)
}

Insgesamt zeigt das Sample eine hohe Zustimmung zum dritten Zukunftsszenario $(M=3,57, S D=0,99)$. Der Wahrnehmung der Befragten zufolge, wird dieses Szenario ebenfalls in den nächsten zehn Jahren relevant werden. Etwa 56,5\% drückten Zustimmung oder gar starke Zustimmung zum MyCurriculum Modell aus (Zustimmungsindex $A$ ) (siehe Abbildung 59). 


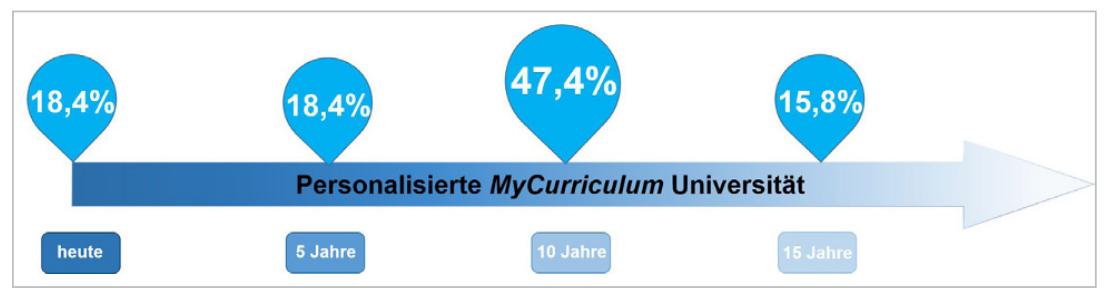

Abb. 59 Geschätzte Zeitdauer für die zunehmende Wichtigkeit des MyCurriculum Szenarios $(\mathrm{N}=46)$

Die Diskussion der Expertinnen und Experten zeigt, dass dieses Szenario in Bezug auf Studierfähigkeit anspruchsvoll ist und einen erhöhten Unterstützungsbedarf auf Seiten der Studierenden nach sich ziehen würde. Einerseits können sich Studierende durch die Flexibilisierung auf Fächer fokussieren, die für sie besonders interessant sind. Andererseits kann in Frage gestellt werden, ob Studierende schon bereit dafür sind zu entscheiden, was nicht nur interessant, sondern auch wichtig zu wissen ist. Hochschulen müssen daher ganz neue Formen, und eine andere Expertise der Unterstützung in Form von Coaching und Mentoring entwickeln. Erste Erfahrungen mit dem MyCurriculum Ansatz zeigen, dass Hochschulen Studierende auffordern, ihre Vorschläge zum Studium zusammenzustellen, und dann einem beratenden Curriculumgremium vorzulegen, welches dann gemeinsam mit dem beziehungsweise der Studierenden berät, wie geeignet der geplante Studienpfad ist.

Darüber hinaus sei - so die Meinung der Befragten der NextSkills Studie - davon auszugehen, dass der Grad des Personalisierungspotentials abhängig vom jeweiligen Studienfeld, nationalen Kontexten des Hochschulsystems sowie Traditionen und den Studierenden selbst sei:

"I believe that there is room for personal learning paths for professionals and skilled workers, who wish to upgrade or diversify their work. However, younger students don't always have much of an idea on their personal study needs, but professionals in the field can better make these choices." (Teilnehmer an NextSkills Delphi-Studie)

Eine zusätzliche Herausforderung ist die dadurch zunehmende Diversität der Abschlüsse, die sich in den personalisierten Curricula reflektieren würde. Daher müssten weitere Mechanismen eingeführt werden, die sicherstellen, dass die Studienerfahrungen sinnvoll dokumentiert und für Dritte verständlich dargestellt sowie Qualitätskonzepte für individualisierte Studienverläufe entwickelt werden können. 
Die Geschwindigkeit der Realisierung für dieses Szenario wird in Abhängigkeit von verschiedenen Faktoren gesehen: Die technologische Infrastruktur, Beratungs- und Betreuungskompetenzen der Lehrenden - die zu Mentorinnen, Mentoren, Begleiterinnen und Begleitern ausgebildet werden müssen - sowie ein hohes Maß an Autonomie und Verantwortungsübernahme von Studierendenseite. Eine steigende Zahl an Studierenden, bei gleichzeitig geringer werdender staatlicher Grundfinanzierung der Hochschulen werden als besondere Herausforderungen im Kontext des MyCurriculum Szenarios benannt. Zudem betonten die Befragten die Abhängigkeit der Realisierbarkeit des Szenarios von der Fähigkeit zum selbstgesteuerten Lernen der Studierenden.

\section{3.2.4 Szenario 4: Die Lifelong Learning University}

In diesem Szenario wird lebenslange akademische Bildung als genauso wichtig erachtet wie initiale Hochschulbildung zu Beginn der Berufsphase. Berufstätige machen dabei den Großteil der Studierenden aus, die ihr Modul-Portfolio entsprechend ihrer persönlichen Skill-Bedürfnisse und Kompetenz-Bedarfe mit einem hohen Maß an Autonomie über ihren Lebensverlauf hinweg selbstständig und je nach Anforderungen auswählen. Institutionen bieten daher auch alternative Zertifizierungsverfahren, wie beispielsweise Microcredentials an, welche die Studierenden individuell und ihren eigenen Interessen und Bedürfnissen entsprechend erwerben können. Anerkennung bereits erbrachter Studienleistungen und praktischer Erfahrungen erlauben es in diesem Szenario, dass flexibel zwischen verschiedenen Anbietern von Hochschulbildung hin- und hergewechselt werden kann. Diese ermöglichen es den Studierenden, frühere Lernerfahrungen in Form von umfassenderen Zertifikaten auszuweisen.

Im Szenario werden vier wesentliche Dimensionen angesprochen, die für dessen Realisierung wesentlich weiterentwickelt werden müssten: 
- Durchlässigkeit: Die Schaffung von Zugangswegen für beruflich Qualifizierte sowie die Anerkennung von Kompetenz aus akademischen und nicht akademischen Vorqualifikationsphasen müsste garantiert werden. In Deutschland machen Landesgesetzgebungen und Hochschulpraxis hierbei starke Fortschritte, bleiben jedoch hinter der im Szenario formulierten Gleichwertigkeit lebenslanger und initialer akademischer Bildung zurück.

- Studienorganisation: Hier stehen insbesondere die Schaffung von flexiblen berufsbegleitenden Studienformaten sowie von Teilzeitmodellen und auch von Short Courses, Micro- und Nanodegree-Formaten im Mittelpunkt. Zudem erfordert die Organisation des Studiums von Hochschulen über die Lebenspanne hinweg, das Schaffen von entsprechenden Verwaltungsabläufen sowie von lehrorganisatorischen Abläufen für entsprechende Angebote.

- Studienformate: Geeignete flexible Studienformate, sowohl im Hinblick auf die sinnvolle Integration digitaler Medien in die Lehre als auch hinsichtlich flexibler Zeiten und räumlicher Möglichkeiten sowie didaktisch in Bezug auf die Zielgruppe berufstätiger Erwachsener werden notwendig.

- Wissenschaftliche Weiterbildung: Die Schaffung von gesetzlichen und hochschuladministrativen Rahmenbedingungen sowie Strategien, in denen Hochschullehrende Lehrangebote im Bereich lebenslangen Lernens im Rahmen ihrer Tätigkeit und Deputatsverpflichtung gestalten können.

Die lebenslang Studierenden stellen eine zunehmend wachsende Zielgruppe für Hochschulen dar, die in diesem Szenario zahlenmäßig schließlich noch zu den bereits existierenden, traditionellen Zielgruppen hinzukommen. Eine interessante aber bislang in Deutschland noch nicht umgesetzte Idee ist die Einführung einer lebenslangen Matrikelnummer, die es Studierenden zumindest schon einmal verwaltungstechnisch vereinfachen würde, Studienangebote im Lebensverlauf auch nach dem offiziellen Ende eines ersten oder zweiten Studienzyklus (Bachelor- oder Masterstudium) wahrzunehmen. Hochschulbildung würde so von ihrem momentanen up front Modus zu einer neuen Form des nahtlosen lebenslangen akademischen Lernens übergehen. Dabei ist eine erste akademische Qualifikationsphase, die dem derzeitigen Bachelor- oder dem Masterstudium entspricht, nicht ausgeschlossen. Diese würde jedoch lediglich den Auftakt für den weiteren akademischen Lebenslernweg bilden. Studierende würden ihr Modulportfolio in diesem Szenario mit hoher Autonomie und entsprechend ihrer persönlichen, beziehungsweise professionellen Skill-Bedarfe und Kompetenzanforderungen ihrer jeweiligen Lebensphase wählen. Hochschulen würden hingegen portable Mikrozertifikate anbieten. Diese könnten Studierende sich dann - je nach ihren eigenen Präferenzen - kumulativ oder komplementär für ein individuelles Abschlussprofil zusammenstellen. 
Das vierte Szenario erhielt die höchsten Zustimmungswerte der Teilnehmerinnen und Teilnehmer des NextSkills Delphis: 71,7\% des Samples gaben hierzu eine (starke) Zustimmung (Zustimmungsindex $A$ ) an (siehe Abbildung 60). Die Befragten unterstrichen, dass Individuen, um im heutigen vom Wettbewerb bestimmten global village erfolgreich sein zu können, einen nie vorher dagewesenen Bedarf an lebenslangen akademischen Lernangeboten hätten.

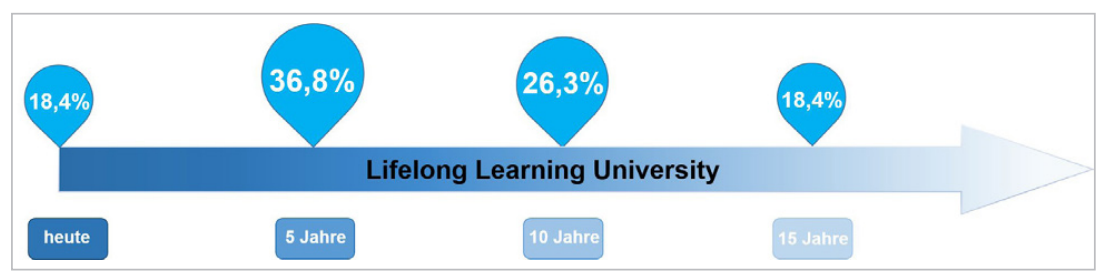

Abb. 60 Geschätzte Zeitdauer für die zunehmende Wichtigkeit des lebenslangen akademischen Lernens $(\mathrm{N}=46)$

Die Zielgruppe der lebenslangen akademischen Lernenden hat - von einzelnen Ausnahmen abgesehen - bislang eher am Rande der strategischen Bemühungen von Hochschulen um den Ausbau ihrer Studienangebote gestanden, die sich primär am Typus der Normalstudierenden (Studienaufnahme direkt oder kurz nach Erwerb der Studienberechtigung ohne vorherige berufliche Qualifikation, Studium in Vollzeit-/Präsenzmodus) orientieren. Es sind vor allem drei gesellschaftliche Dynamiken gewesen, welche das vierte Szenario befeuern:

1. Der demografische Wandel,

2. der von vielen Arbeitsmarktexpertinnen und -experten prognostizierte Fachkräftebedarf und

3. die Forderung nach einer größeren Durchlässigkeit zwischen beruflicher und akademischer Bildung.

In Bezug auf die Einschätzung der Zeitspanne, in der das Szenario realisiert werden kann, ergibt sich eine zur Wichtigkeit leicht konträre Einschätzung. Während es unter den Expertinnen und Experten eine stark zustimmende Einschätzung bezüglich der Relevanz gibt, wird das Szenario in dieser deutlich und pointiert formulierten Variante (lebenslanges akademisches Lernen würde als genauso wichtige wie das derzeitige Modell der Hochschulbildung angesehen) als nicht unmittelbar und heute schon realisierbar eingeschätzt. Die Mehrheit der Expertinnen und Exper- 
ten geht davon aus, dass dieses Szenario bereits in den nächsten fünf Jahren an Relevanz gewinnen wird (55,2\%). Jedoch schätzt jeder vierte Befragte die time of adoption auf circa zehn Jahre sowie jeder Fünfte auf fünfzehn Jahre. Die Befragten sehen in Bezug auf das Szenario eher einen evolutionären Pfad der Veränderung als realistisch an. Dieser verläuft nach ihrer Ansicht zunächst über eine stärkere Öffnung der Hochschulen für berufstätige lebenslange Lernende. Damit einher ginge zudem auch, dass Hochschulen künftig einen stärkeren Fokus darauf richten würden, intensive Beziehungen mit ihren Alumni zu unterhalten, die sich nach der Graduierung wieder an ihre alma mater wenden würden, wann immer sich für sie in ihrem Berufsleben akademischer Weiterbildungsbedarf ergäbe.

Herausforderungen in Bezug auf das Szenario entstehen dadurch, dass ein tatsächlicher Paradigmenwandel in Bezug auf akademische Bildung vollzogen werden muss, der auch Auswirkungen auf die bestehenden Studienangebote und Hochschulstrukturen hat. Zusätzlich ist zu berücksichtigen, dass ein Wandel zur lebenslangen akademischen Bildung nicht allein von den Hochschulen gestaltet werden kann, sondern auch von den lernenden Individuen und deren Bereitschaft zur Bildungsbeteilung sowie von den Arbeitgeberinnen und Arbeitgebern abhängt. Diese müssen die Wichtigkeit lebenslangen akademischen Lernens anerkennen und ihren Mitarbeiterinnen und Mitarbeitern entsprechend zusätzlich Lern- und Fortbildungsgelegenheiten zugestehen. 